\title{
Mortality of white South African gold miners
}

\author{
PJ Reid, GK Sluis-Cremer
}

\begin{abstract}
Objectives-This two part study aimed to determine whether there was an excess mortality generally or for some diseases among middle aged white South African gold miners on the Witwatersrand and whether the underground dust exposure of these miners contributed to the development of lung cancer, chronic obstructive pulmonary disease (COPD), or ischaemic heart disease (IHD).
\end{abstract}

Methods-A cohort of 4925 white miners in South Africa, born between 1 January 1916 and 31 December 1930 who were alive and working in the vicinity of Johannesburg on 1 January 1970, then aged between 39 and 54, was followed up for 20 years by which time 2032 had died. Most were gold miners (about $87 \%$ had worked $85 \%$ or more of their shifts in gold mines). Standardised mortality ratios (SMRs) were calculated as percentages of the number of deaths observed in the cohort for a condition as stated on the death certificate divided by the number expected on the basis of concurrent mortality in the reference population (the total age specific white male population of South Africa). A case-control analysis was performed for three diseases (lung cancer, COPD, and IHD), the results of which are presented for those miners in the cohort who had spent at least $85 \%$ of their service on gold mines and had worked at least $15 \%$ of their shifts underground.

Results-The SMR for all causes of death was $129.6 \%$, raised because of excess mortality due to the following causes: lung cancer $(S M R=139 \cdot 8 \%)$, IHD $(124 \cdot 1 \%)$, COPD $(189 \%)$ and cirrhosis of the liver $(155 \cdot 3 \%)$. Smoking was confirmed to be the main risk factor for lung cancer and COPD although cumulative dust exposure was found to increase the risk of COPD in conjunction with smoking. No significant risk of lung cancer resulted from exposure to dust. High blood pressure and smoking were found to increase the risk of IHD, but no association between IHD and the quetelet index (weight/height ${ }^{2}$ ) was found.

Conclusions-The most significant and unexpected finding was the $30 \%$ increase in the SMR for all causes. Very little of this increase could be attributed to mining and the main factor for this was probably the adoption of an unhealthy lifestyle by these men (compared with other South African white men) particularly smoking (86\% were smokers) and alcohol consumption.

\section{(Occup Environ Med 1996;53:11-16)}

Keywords: mortality; gold miners

In South Africa gold miners work in stressful conditions of heat and humidity and are exposed to low levels of dust containing a moderate amount of silica and to low concentrations of radon daughters. They are also exposed to unknown concentrations of other atmospheric pollutants, such as nitrous fumes from the blasting operations and exhaust fumes where diesel engines are used.

This two part study aimed to determine whether there was an excess mortality both generally and for certain diseases among middle aged white South African miners, who were currently working on the gold mines in the East Rand, Central Rand, and West Rand gold mining regions of the Transvaal and also whether work in the gold mines contributed to the development of certain of these diseases. The first part was the calculation of standardised mortality ratios (SMRs) in a cohort of 4925 miners at work on 1 January 1970 , followed up to the end of 1989 , with as a reference population, the total white male population of the Republic of South Africa. The second part was a case-control analysis that followed on from Liddell et $a l^{1}$ and SluisCremer et al. ${ }^{2}$ The results are reported for that proportion of the same cohort who had at least $85 \%$ of their service on gold mines and who had worked underground at least $15 \%$ of their shifts and are concerned with deaths from lung cancer, chronic respiratory diseases, and ischaemic heart disease (IHD). These specific diseases were chosen for the case-control analysis because there was reason to think that service in the gold mines might contribute to the development of these diseases either due to the years of underground service or due to cumulative dust exposure.

\section{Subjects and methods}

The target population was all white gold miners who, at the start of 1970 , were aged 39 to 54 and had attended the Medical Bureau for Occupational Diseases (MBOD) in 1969. They were currently working in gold mines in an area defined as the East Rand-Central Rand-West Rand geographical region.

Every employee of any mine in the Republic of South Africa who works underground or on the surface in occupations with specified risks (defined by law on the basis of the degree of 
dust exposure) is legally required to present himself for an initial medical examination at the MBOD for a certificate of fitness for work in a risky occupation. The miner is also required to renew this certificate of fitness each year by a further medical examination. A miner may also request a compensation examination (discussed later), which is carried out at the MBOD. Those miners who were born between 1 January 1916 and 31 December 1930, who attended the MBOD in 1969, and who were known to be alive on 1 January 1970 , numbered 5062. Of these, however, a total of $137 \mathrm{did}$ not meet the selection criteria-either they were not employed in 1970 in mines in the area defined above; or they had been referred to the MBOD from outside the designated area.

The remaining 4925 miners formed the study cohort. They are known (through estimates from Chamber of Mines records) to be such a high proportion of the men working in the defined area that enumeration must have been virtually complete. ${ }^{3}$

White miners in the Republic of South Africa are particularly mobile and a proportion of those in the cohort have, as well as service in gold mines, had service in other types of mines. The objective in the case-control analysis was to find the effect of factors associated with service, primarily in gold mines, on the three diseases of specific interest. For the purposes of this part of the study we present the analysis only for subjects who had at least $85 \%$ of their service in gold mines as well as at least $15 \%$ underground. The proportion of $85 \%$ was chosen because it was used in the previous study to indicate that each man had a large proportion of his service in gold mines. From data obtained for the case-control component of this study we found that most $(87 \%)$ had $85 \%$ or more of their service in gold mines and $90 \%$ had worked underground for at least $15 \%$ of their service; $80 \%$ fulfilled both criteria.

\section{DATA COLLECTION}

It was possible to establish the vital status of all but 50 members of the cohort on 31 December $1989-20$ years from the date of entry into the cohort. These 50 in the absence of a death certificate or other evidence of death were assumed to be still alive.

Death registration certificates of every person dying in the Republic of South Africa are collected and filed by the Department of the Interior. Each year, they are submitted to the Central Statistical Services where coding clerks allocate to each certificate a rubric (code number) according to the international classification of diseases (ICD). The ICD code number is written on to the death registration certificate and it is then returned to the Department of the Interior for filing. In the first eight years of the study the eighth revision of the ICD code (ICD-8) was used, but thereafter ICD-9 was used. The coding clerks followed the guidelines set out in the World Health Organisation manual for classifying causes of death and allocating code numbers; however, their expertise is limited and they only have information from the death certificate.

Best available information on the cause of each death was also assessed by one of us (GKS-C). This is a considerably more reliable cause of death than that coded on the death certificate for two reasons: the medical knowledge of (GKS-C) is greater than that of the clerks and he had additional information (the clinical notes of the MBOD and the postmortem report).

Under the heading of chronic obstructive pulmonary diseases (COPD) we have grouped together bronchitis, emphysema, asthma, and chronic airways obstruction (ICD codes 490-96), pneumoconiosis due to silica or other dust (ICD codes 500-5) and corpulmonale referred to as pulmonary heart disease (ICD code 416).

\section{METHODS OF ANALYSIS}

Subject-years method

The first analysis used the subject-years method to calculate the SMRs. Subject-years at risk during the study interval were calculated as described by Breslow and Day. ${ }^{4}$ The choice of the reference population, the total white South African male population, was restricted by the availability of mortality figures and population estimates in the Republic of South Africa. The Central Statistical Services for the Republic of South Africa publish annual reports on the numbers of deaths and the death rates by race, sex, age, and cause. Mid-year population estimates for white South African men by quinquennial age groups for the 20 single calendar years of the study interval are also published or can be easily estimated from this information. From these rates and from the relevant person-years in view during the study interval, the age and cause specific expected numbers of deaths were found.

The numbers of deaths observed, cause by cause, from the death registration certificate, were counted by age group and year. The observed (Obs) and expected (Exp) deaths, by cause, were summed over each age group for the 20 years of follow up, and the SMR was obtained as 100 (Obs/Exp). The 95\% confidence intervals (95\% CIs) for the SMRs and $P$ values were evaluated with the Rothman and Boice approximation formulae. ${ }^{45}$ All these calculations were executed by Dbase programs.

Data on smoking are available for all subjects, and from available records it would also have been possible to obtain complete histories of mining employment but it would have been prohibitively laborious to collect such details for the complete cohort. Thus there was no study of such factors within the first analysis.

Case-control analysis

The second analysis was of the case-control form, within the cohort, after the method of 
Liddell $e t a l^{1}$ and Sluis-Cremer et $a l^{2}$ for the three diseases of specific interest, lung cancer (159 deaths), COPD (160 deaths), and IHD (676 deaths), all according to the best available information.

For each case, with cause of death assessed according to the best available information, a set of controls was selected strictly at random from among all those miners born in the same year as the case and who survived the case. No other matching for risk factors was performed. It was possible that a case could be selected as a control ${ }^{46}$ for another case-control set if he had outlived the case for that set, and was born in the same year. For practical reasons with the randomisation process to select controls, a subject once selected as a disease control was excluded from reselection as a control for other cases with the same disease, but could be chosen as a control for a case that had died from one of the other diseases studied.

Two controls were selected for each death from lung cancer or COPD but, because there were so many deaths due to IHD it was deemed sufficient to only select one control per case of IHD.

The following possible risk factors were investigated in the case-control analysis.

Smoking-Smoking was considered a risk factor for all three diseases. Smoking, which included cigarettes, cigars, or pipes smoked was converted to the number of cigarettes smoked a day with standard conversion factors ( 1 cigar $=5$ cigarettes, $25 \mathrm{~g}$ pipe-tobacco $=$ 11 cigarettes). The amount smoked was recorded for the years 1960-90 by five year intervals, but was coded as missing if the person had died or the information was not obtained. We used an average of the amount smoked per day in $1960-90$ as the quantitative variable to represent smoking exposure. (Note that missing data points are ignored by the SAS function mean that was used to do this.)

Mining service - Two indicators of mining service were obtained: total duration of underground service and duration of cumulative dust exposure expressed in $y-\mathrm{mg} / \mathrm{m}^{3}$. The Chamber of Mines' employment history records give shifts worked by calendar period and occupation. Seven occupational categories were identified according to the degree of dust experienced within these occupations. These included dusty occupations both on the surface and underground. The number of shifts worked was extracted for all members of each case-control set over the period from the date on which the subject's service started until either death or the end of the record. The choice of which end point to use in the calculation of the cumulative dust exposure was given much thought as the wrong choice results in bias when the relative risk of exposure is calculated by the conditional logistic regression analysis. We eventually chose as the end point a date five years before the death of the case in each case-control set.

Our choice of a lag period of five years is arbitrary but just as it is impossible for a case to accumulate any dust exposure after death it is also likely that he would not accumulate much dust exposure for the period of illness preceding death due to lung cancer, COPD, or IHD, so the introduction of some lag period is reasonable. As a point of interest the results were calculated for other end points-for example, the start of the study, which is unbiased, and the death of the subject, which is biased-but these results are not presented.

The previous study used as the end point for cumulative dust exposure the date of the last dusty shift worked by the case in the casecontrol set, and for underground years of service the date of the last underground shift worked by the case, which effectively censors the exposure variables of the controls and so is biased towards finding an increased exposure in the case-that is, the relative risk would be calculated higher than it should be.

The number of shifts was divided by 240 to obtain a measurement of mining service in years. This figure was obtained from the data when they occurred so that dates and shifts were both coded.

Dust exposures estimated by thermal precipitator count of respirable mass after acid treatment are used to calculate dust exposure in $\mathrm{h}-\mathrm{mg} / \mathrm{m}^{3}$ per shift. Multiplying the number of shifts in any of the seven occupational categories by this dust exposure in $\mathrm{h}-\mathrm{mg} / \mathrm{m}^{3}$ per shift as reported by $\mathrm{Beadle}^{7}$ gives the cumulative dust exposure in $\mathrm{h}-\mathrm{mg} / \mathrm{m}^{3}$.

We divide this by 1920 hours/year (240 shifts/year at 8 hours/shift) to get the cumulative dust exposure expressed in $\mathrm{y}-\mathrm{mg} / \mathrm{m}^{3}$ (the mass of respirable dust (mg) after acid treatment per cubic metre of air, multiplied by the hours exposed per shift, divided by the average working hours in a year).

The dust exposure for each of the six dusty categories in gold mines (where $99 \%$ of shifts in the case-control study were worked) was assessed to be:

high dust shift (shaft sinker, developer) $=$ $2 \cdot 14 \mathrm{~h}-\mathrm{mg} / \mathrm{m}^{3}=0.00115 \mathrm{y}-\mathrm{mg} / \mathrm{m}^{3}$ medium dust shift (stoper) $=2 / 3$ high dust shift $=1.43 \mathrm{~h}-\mathrm{mg} / \mathrm{m}^{3}=0.00074 \mathrm{y}-\mathrm{mg} / \mathrm{m}^{3}$ medium dust shift (shift boss, trammer, etc) $=1 / 2$ high dust shift $=1.07$ h-mg $/ \mathrm{m}^{3}=$ $0.00056 \mathrm{y}-\mathrm{mg} / \mathrm{m}^{3}$

low dust shift (artisans, surveyors, etc) $=1 / 3$ high dust shift $=0.71 \mathrm{~h}-\mathrm{mg} / \mathrm{m}^{3}=0.00037 \mathrm{y}-$ $\mathrm{mg} / \mathrm{m}^{3}$

surface dust shift $=1 / 4$ high dust shift $=0.53$

$\mathrm{h}-\mathrm{mg} / \mathrm{m}^{3}=0.00029 \mathrm{y}-\mathrm{mg} / \mathrm{m}^{3}$

surface non-dusty shift $=0 \mathrm{~h}-\mathrm{mg} / \mathrm{m}^{3}$

These exposures and units were in broad agreement with the published data in the papers by Beadle ${ }^{7}$ and Page-Shipp and Harris. ${ }^{8}$

The seventh category was for low dust risk in non-gold mines where the dust exposure was assessed as $1^{\prime} / 6$ that of high dust exposure in a gold mine $\left(0.36 \mathrm{~h}-\mathrm{mg} / \mathrm{m}^{3} / \mathrm{shift}\right)$.

The effects of both cumulative dust exposure and total duration of underground service were analysed separately, the total duration of underground service provided a surrogate measurement of the miner's lifetime exposure to the low concentrations of radon and 


\begin{tabular}{|c|c|c|c|c|c|c|}
\hline \multirow[b]{2}{*}{ Cause of death } & \multirow{2}{*}{$\begin{array}{l}\text { 9th rev'n } \\
\text { ICD codes }\end{array}$} & \multicolumn{2}{|l|}{ Deaths } & \multirow[b]{2}{*}{$S M R$} & \multirow[b]{2}{*}{$(95 \% C I)$} & \multirow[b]{2}{*}{ Pvalue } \\
\hline & & $O B S$ & $E X P$ & & & \\
\hline Total deaths & 0-999 & 2032 & 1568 & $129 \cdot 6$ & $(124 \cdot 0-135 \cdot 4)$ & $<0.0001$ \\
\hline Cancer & $140-239$ & 341 & $308 \cdot 77$ & $110 \cdot 4$ & $(99 \cdot 0-122 \cdot 8)$ & NS \\
\hline Lung cancer & 162 & 143 & $102 \cdot 32$ & $139 \cdot 8$ & $(117 \cdot 8-164 \cdot 6)$ & $<0.0005$ \\
\hline Stomach cancer & 151 & 29 & $24 \cdot 45$ & $118 \cdot 6$ & $(79 \cdot 4-170 \cdot 4)$ & NS \\
\hline Mesothelioma & 509 & 4 & $3 \cdot 34$ & $119 \cdot 8$ & $(32 \cdot 2-306 \cdot 6)$ & NS \\
\hline Tuberculosis & $10-19$ & 22 & $7 \cdot 18$ & $306 \cdot 4$ & $(192 \cdot 0-463.9)$ & $<0.0001$ \\
\hline Pulmonary tuberculosis & 11 & 20 & $6 \cdot 75$ & $295 \cdot 3$ & $(180 \cdot 9-457 \cdot 6)$ & $<0.0001$ \\
\hline Pneumonia & $480-486$ & 68 & $46 \cdot 71$ & $145 \cdot 6$ & $(113 \cdot 0-184 \cdot 6)$ & $<0.005$ \\
\hline Bronchitis & $490-491$ & 36 & $13 \cdot 81$ & $260 \cdot 7$ & $(182 \cdot 6-360 \cdot 9)$ & $<0.0001$ \\
\hline Emphysema & 492 & 33 & $29 \cdot 81$ & $110 \cdot 7$ & $(76 \cdot 2-155 \cdot 5)$ & NS \\
\hline Bronchitis emphysema & $490-492$ & 69 & $43 \cdot 64$ & $158 \cdot 1$ & $(123 \cdot 0-200 \cdot 1)$ & $<0.0005$ \\
\hline Asthma & 493 & 13 & $11 \cdot 58$ & $112 \cdot 3$ & $(59 \cdot 7-192 \cdot 0)$ & NS \\
\hline Bronchiectasis & 494 & 0 & $0 \cdot 70$ & 0.0 & $(0 \cdot 0-524 \cdot 0)$ & NS \\
\hline Chronic obstructive airways & 496 & 138 & $85 \cdot 78$ & $160 \cdot 9$ & $(135 \cdot 2-190 \cdot 1)$ & $<0.0001$ \\
\hline Pneumoconiosis silicosis asbestosis & $500-505$ & 16 & $0 \cdot 75$ & 2133 & $(1219-3465)$ & $<0.0001$ \\
\hline Cor-pulmonale & 416 & 22 & $6 \cdot 62$ & $332 \cdot 3$ & $(208 \cdot 2-503 \cdot 2)$ & $<0.0001$ \\
\hline COPD & $416490-505$ & 176 & $93 \cdot 14$ & $189 \cdot 0$ & $(162 \cdot 1-219 \cdot 0)$ & $<0.0001$ \\
\hline Ischaemic heart disease & $410-414$ & 687 & $553 \cdot 68$ & $124 \cdot 1$ & $(115 \cdot 0-133 \cdot 7)$ & $<0.0001$ \\
\hline Hypertensive disease & $400-405$ & 17 & $17 \cdot 87$ & $95 \cdot 1$ & $(55 \cdot 4-152 \cdot 3)$ & NS \\
\hline Cerebrovascular disease & $430-438$ & 109 & $100 \cdot 22$ & $108 \cdot 8$ & $(89 \cdot 3-131 \cdot 2)$ & NS \\
\hline Peptic ulcer disease & $531-535$ & 21 & $10 \cdot 87$ & $193 \cdot 2$ & $(119 \cdot 5-295 \cdot 3)$ & $<0.01$ \\
\hline Liver cirrhosis & 609571 & 45 & $28 \cdot 97$ & $155 \cdot 3$ & $(113 \cdot 3-207 \cdot 9)$ & $<0.01$ \\
\hline Alcoholic liver cirrhosis & 609 & 11 & $5 \cdot 85$ & $188 \cdot 0$ & $(93 \cdot 7-336 \cdot 5)$ & NS \\
\hline Non-alcoholic liver cirrhosis & 571 & 34 & $23 \cdot 08$ & $147 \cdot 3$ & $(102 \cdot 0-205 \cdot 9)$ & $<0.05$ \\
\hline Renal failure & $580-589$ & 24 & $14 \cdot 68$ & $163 \cdot 5$ & $(104 \cdot 7-243 \cdot 3)$ & $<0.05$ \\
\hline Unknown cause of death & 799 & 71 & $52 \cdot 20$ & $136 \cdot 0$ & $(106 \cdot 2-171 \cdot 6)$ & $<0.05$ \\
\hline Motor vehicle accident & $810-819$ & 55 & $38 \cdot 87$ & $141 \cdot 5$ & $(106 \cdot 6-184 \cdot 2)$ & $<0.05$ \\
\hline Suicide & $950-959979$ & 45 & $33 \cdot 54$ & $134 \cdot 2$ & $(97 \cdot 9-179 \cdot 5)$ & NS \\
\hline
\end{tabular}

There were 464 excess deaths.
More than half (257) accounted for by IHD, lung cancer, and COPD

More than half (257) accounted for by IHD, lung cancer, and COPD.
Another 35 excess deaths from unknown causes and liver cirrhosis.

Another 35 excess deaths from unknown causes and
SMR (expressed as percentage) $=100^{\star} \mathrm{Obs} / \mathrm{Exp}$.

95\% CIs calculated with Rothman and Boice formulas $a=0.05$.

That is, SMR lower limit $=\operatorname{SMR}\left(1-1 / 9 \mathrm{Obs}^{-1}-1 / 3\left(Z_{a / 2}=1.96\right) \mathrm{Obs}^{-1}\right)$

SMR upper limit $\left.=\operatorname{SMR}(1-1 / 9 \text { Obs }+1)^{-1}+1 / 3(Z a / 2)(\text { Obs }+1)^{-1}\right)^{3}$
$P$ value calculated backwards from formulas solving for " $a$ " so either $=100$

its daughters found in South African gold mines.

\section{BLOOD PRESSURE AND QUETELET INDEX}

These two additional risk factors were considered for deaths from ischaemic heart disease. Blood pressure measurements were recorded in the clinical notes at the start of the study (1969) and at later annual examinations. We captured for analysis the values in 1969 and the last two values from the notes. We present the results of the risk analysis and regression for the 1969 values.

For the quetelet index of obesity, weight (W) has been recorded at the same time points, but height $(\mathrm{H})$ was recorded only at the 1969 examination. We again used the values recorded at the start of the study in the calculation of the index $\left(\mathrm{W} / \mathrm{H}^{2}\right)$, and subsequent risk analysis.

To model the effects of these variables on the risk of disease, we used the statistics package GLIM (general linear interactive models) with the pecan option for conditional logistic regression. The SAS procedure Nlin and the Macro \%Condlog also perform this conditional regression and give the same figures for the relative risk but calculate a very conservative SEM for this risk which is larger than that obtained by other methods. For dichotomous variables the SAS procedure Freq can calculate the Mantel-Hansel odds ratio which is an estimate of the relative risk. For matching case-control pairs the SAS procedure Logist can be used to perform conditional analysis.

\section{Results}

STANDARDISED MORTALITY RATIOS (SMRS)

Of the 4925 miners in the cohort, 2892
$(58.7 \%)$ survived into 1990 . The deaths numbered 2032, compared with 1568 expected, leading to an SMR of 129.6 substantially in excess of 100 .

Table 1 shows the observed numbers of deaths in the cohort from selected causes and the numbers expected, the SMRs, and the corresponding $95 \%$ CIs. Judged by these CIs there was significant excess mortality, compared with the reference population of all white men in the Republic of South Africa, for lung cancer, COPD, IHD, cirrhosis of the liver, and kidney failure. It must be made clear that these figures are based on the causes of death given on the death certificates to make valid comparisons with the deaths in the reference population.

\section{RESULTS OF THE CASE-CONTROL STUDY}

Table 2 shows the results of the case-control studies. Risk factors were found by matched case-control studies for lung cancer (ICD = 162), COPD (ICD-9 = 416 + 490-505), and IHD (ICD 410-414), among white gold miners (aged $39-54$ in 1970 with at least $85 \%$ of service on gold mines and at least $15 \%$ of shifts underground).

These restrictions resulted in a population in which over $99 \%$ of the shifts worked were in gold mines and $88 \%$ were underground. Table 2 shows the well established result that smoking cigarettes increases the risk of dying from lung cancer, COPD, or IHD, and that these risks increase the more cigarettes are smoked per day.

Furthermore this study shows the risk from smoking for COPD to be almost twice that for lung cancer.

Not a single case in this study of death due to COPD occurred in a non-smoker, indicating 
Table 2 Relative risks

\begin{tabular}{|c|c|c|c|c|c|c|}
\hline \multirow[b]{2}{*}{ Relative risks } & \multicolumn{2}{|l|}{ Lung cancer } & \multicolumn{2}{|l|}{$C O P D$} & \multicolumn{2}{|l|}{$I H D$} \\
\hline & Risk $(95 \% C I)$ & Pvalue & $\operatorname{Risk}(95 \% \mathrm{CI})$ & P value & Risk $(95 \%$ CI) & P value \\
\hline Amount smoked (packs of $20 /$ day) & $2 \cdot 41(1 \cdot 4-4 \cdot 2)$ & 0.002 & $4 \cdot 30(2 \cdot 2-8 \cdot 3)$ & $<0.001$ & $1 \cdot 67(1 \cdot 2-2 \cdot 4)$ & $0 \cdot 004$ \\
\hline $\begin{array}{l}\text { Cumulative } 5 \mathrm{y} \text { before case death }\left(\mathrm{y}-\mathrm{mg} / \mathrm{m}^{3}\right) \\
\text { (and after entering smoking to model) }\end{array}$ & $\begin{array}{l}1.08(0.94-1 \cdot 2) \\
1 \cdot 12(0.97-1 \cdot 3)\end{array}$ & $\begin{array}{l}0 \cdot 27 \\
0 \cdot 13\end{array}$ & $\begin{array}{l}1.23(1.0-1 \cdot 5) \\
1.20(1.0-1.4)\end{array}$ & $\begin{array}{l}0.013 \\
0.043\end{array}$ & & \\
\hline Underground decades (2400 shifts) 5 y & $0.99(0.77-1 \cdot 3)$ & 0.92 & $1 \cdot 10(0 \cdot 8-1 \cdot 5)$ & $0 \cdot 51$ & $0.97(0 \cdot 83-1 \cdot 1)$ & $0 \cdot 749$ \\
\hline (and after entering smoking to model) & $1 \cdot 00(0 \cdot 78-1 \cdot 3)$ & 0.99 & $1.02(0 \cdot 8-1 \cdot 4)$ & $0 \cdot 87$ & $0.98(0.83-1 \cdot 2)$ & $0 \cdot 831$ \\
\hline $\begin{array}{l}\text { Systolic BP (1970) (10 mm Hg) } \\
\text { (and after entering smoking to model) }\end{array}$ & & & & & $\begin{array}{l}1 \cdot 14(1 \cdot 0-1 \cdot 2) \\
1 \cdot 15(1 \cdot 1-1 \cdot 2)\end{array}$ & $\begin{array}{l}0.002 \\
0.001\end{array}$ \\
\hline $\begin{array}{l}\text { Diastolic BP (1970) (10 mm Hg) } \\
\quad \text { (and after entering smoking to model) }\end{array}$ & & & & & $\begin{array}{l}1 \cdot 27(1 \cdot 1-1 \cdot 4) \\
1 \cdot 29(1 \cdot 1-1 \cdot 5)\end{array}$ & $\begin{array}{l}0.001 \\
0.001\end{array}$ \\
\hline $\begin{array}{l}\text { Quetelet index }(1970)\left(10 \mathrm{~kg} / \mathrm{m}^{2}\right) \\
\text { (and after entering smoking to model) }\end{array}$ & & & & & $\begin{array}{l}1 \cdot 14(0 \cdot 77-1 \cdot 7) \\
1 \cdot 20(0 \cdot 80-1 \cdot 8)\end{array}$ & $\begin{array}{l}0 \cdot 511 \\
0 \cdot 381\end{array}$ \\
\hline Case control sets (n) & 130 & & 127 & & 484 & \\
\hline Deaths from death certificate & 143 & & 176 & & 687 & \\
\hline Deaths from best available evidence & 159 & & 160 & & 676 & \\
\hline $\begin{array}{l}\text { SMR for } 1 \text { January } 1970-31 \text { December } 89 \\
(n=4925)\end{array}$ & $139 \cdot 8(118-165)$ & & $189(162-219)$ & & $124 \cdot 1(115-134)$ & \\
\hline
\end{tabular}

that in this study dust alone was never sufficient to cause COPD but could do so in conjunction with smoking. There were only four non-smokers who developed lung cancer one of whom was a boilermaker with established asbestos exposure. It had been suggested by one of us (GKS-C) that miners with asbestos exposure should be excluded from the study. This would have been difficult as from the design of the study we only obtained the full work history late in the analysis and only discovered details of the work done by the miner in a retrospective examination of the clinical notes.

Underground service only was not shown to increase the risk of lung cancer, COPD, or IHD.

Dust was not shown to increase the risk of lung cancer.

A high blood pressure (either systolic or diastolic) was associated with an increased risk

Table 3 SMR study: reliability of the ICD codes

\begin{tabular}{|c|c|c|c|c|}
\hline \multirow[b]{2}{*}{ Cause of death } & \multirow[b]{2}{*}{$I C D-9$} & \multirow[b]{2}{*}{ Deaths } & \multicolumn{2}{|c|}{ Death certificate code } \\
\hline & & & $\%$ wrong & $\%$ missed \\
\hline Total deaths & 0-999 & 2032 & $\mathbf{2 4 \cdot 3}$ & \\
\hline Cancer & $140-239$ & 341 & $5 \cdot 3$ & 13.9 \\
\hline Lung cancer & 162 & 143 & $11 \cdot 9$ & $20 \cdot 8$ \\
\hline Stomach cancer & 151 & 29 & 6.9 & $10 \cdot 0$ \\
\hline Mesothelioma & 509 & 4 & $0 \cdot 0$ & $33 \cdot 3$ \\
\hline Tubrculosis & 10-19 & 22 & $68 \cdot 2$ & $53 \cdot 3$ \\
\hline Pulmonary tuberculosis & 11 & 20 & $65 \cdot 0$ & $30 \cdot 0$ \\
\hline Pneumonia & $480-486$ & 68 & $48 \cdot 5$ & $20 \cdot 5$ \\
\hline Bronchitis & $490-491$ & 36 & $47 \cdot 2$ & 0.0 \\
\hline Emphysema & 492 & 33 & $27 \cdot 3$ & $35 \cdot 1$ \\
\hline Bronchitis emphysema & $490-492$ & 69 & $36 \cdot 2$ & $21 \cdot 4$ \\
\hline Asthma & 493 & 13 & $69 \cdot 2$ & $20 \cdot 0$ \\
\hline COPD & $416490-505$ & 176 & $32 \cdot 7$ & $25 \cdot 5$ \\
\hline Ischaemic heart disease & $410-414$ & 687 & $\mathbf{8 \cdot 0}$ & $6 \cdot 5$ \\
\hline Hypertensive disease & $400-405$ & 17 & $29 \cdot 4$ & $20 \cdot 0$ \\
\hline Cerebrovascular disease & $430-438$ & 109 & $20 \cdot 2$ & $9 \cdot 4$ \\
\hline Peptic ulcer disease & $531-535$ & 21 & $33 \cdot 3$ & $36 \cdot 4$ \\
\hline Liver cirrhosis & 609571 & 45 & $6 \cdot 7$ & $32 \cdot 3$ \\
\hline Alcoholic liver cirrhosis & 609 & 11 & $36 \cdot 4$ & $75 \cdot 0$ \\
\hline Non alcoholic liver cirrhosis & 571 & 34 & $61 \cdot 8$ & $61 \cdot 8$ \\
\hline Renal failure & $580-589$ & 24 & $16 \cdot 7$ & $39 \cdot 4$ \\
\hline Motor vehicle accident & $810-819$ & 55 & 1.8 & 0.0 \\
\hline Suicide & $950-959979$ & 45 & $4 \cdot 4$ & 0.0 \\
\hline Diabetes & 250 & 22 & $13 \cdot 6$ & $24 \cdot 0$ \\
\hline Pulmonary embolus & 415 & 29 & $22 \cdot 2$ & $72 \cdot 0$ \\
\hline
\end{tabular}

$\%$ Wrong = percentage of deaths coded as due to a cause on death certificate that actually died from some other cause. This is equivalent to a false positive rate.

$\%$ Miss = percentage of deaths due to a cause but coded as due to a different cause on death certificate. This is equivalent to a false negative rate. of IHD, but surprisingly we could not show any link between our indicator for obesity (the quetelet index) and IHD.

The typical gold miner of this study serves 27 years underground and was exposed to $3 \cdot 7$ $\mathrm{y}-\mathrm{mg} / \mathrm{m}^{3}=7104 \mathrm{~h}-\mathrm{mg} / \mathrm{m}^{3}$ (after acid treatment). This dust gave him a risk for COPD of $1 \cdot 2^{3 \cdot 7}=2 \cdot 0$ if he smoked (roughly equivalent to smoking $1 / 2$ a pack a day). Another interpretation is that the average underground miner is at twice the risk of COPD than the rest of the population from smoking.

\section{Discussion}

This cohort of miners differs from the control population in that each miner on engagement (normally at the age of 18) underwent a medical examination that excluded men in obviously poor health or who are notably overweight or underweight. One would thus expect a healthy worker effect in the SMR analysis. This is patently not the case as the SMR for all causes of diseases was almost $30 \%$ more than in the control population. The previous study ${ }^{1}$ also found this effect on part of this cohort (3971 miners) over eight years from 1970-8. The calculated SMR then was $117 \cdot 6 \%$. In the full cohort of 4925 miners over the same years the SMR was calculated to be $124 \%$.

Possible reasons for the excessive death rate were discussed in the paper by Wyndham et $a l .^{3}$ For example, a bias towards ill health could have been introduced possibly by the selection of the cohort as people who attended the MBOD. More interestingly by the adoption of certain unhealthy behaviours by these miners relative to the control population, in particular excessive smoking and excessive alcohol intake. The interaction between these unhealthy habits and the mining environment may be the cause of ill health-for example, they may only use alcohol to the same extent as others but may have more accidents due to the presence of heavy machinery at work, or likewise there may possibly be a similar interaction between smoking and dust causing 
COPD, or between stress and smoking and IHD.

To support the argument that unhealthy lifestyles or habits are at least a major factor for the high SMRs is the fact that the three biggest known contributing diseases to this excessive death rate were IHD, COPD, and lung cancer, all of which are well known to be caused or exacerbated by smoking. This fact has been established yet again by the case-control studies done here. To further support the argument the fourth biggest contributor to these excess deaths is cirrhosis of the liver which of course is associated with excessive alcohol consumption. If one then takes into account the unavoidable dangers of underground mine work shown by increases in deaths due to compensatable diseases like tuberculosis, silicosis, mesothelioma, and mine accidents, and then also takes into account the emotional stress of mine work hinted at by excessive suicide and deaths from peptic ulcers, we will have gone a long way towards explaining this $30 \%$ excess in the death rate.

That these miners are heavy smokers is beyond question, $86 \%$ smoked at some time of their lives and most of these smokers admitted to having averaged a pack a day (the average smoked varied over the years between 16 and 17 a day). Is this greatly in excess of average cigarette consumption for the rest of South African white males? We have not been able to obtain data to confirm or refute this suspicion. If, as we think, this smoking was really the major cause of the greatly increased SMRs found in this study it would be a major indictment of the habit (as if it needs more) and would lend credence to the suggestion that an antismoking campaign should be targeted at this population.

So much for the usual medical diatribe against smoking; what of dust? This study found that dust exposure during underground gold mining does probably contribute towards the development of COPD. This effect was small and our interpretation is that a further insult was needed to create disease and disability; this further insult here was smoking.

As for exposure to radon daughters, this study showed no direct relation between underground mining and lung cancer and we may conclude that it was either not a concern in these mines or that the effect was so small compared with cigarette smoking that it was not demonstrable. Of course a count of underground shifts may be a very poor surrogate for exposure to radon and this may be a reason for not finding a relation between this radiation exposure and lung cancer. We are planning studies with more detailed data on exposure to radiation to further investigate its relation with mining and lung cancer.

Obesity as measured by the quetelet index was found to be a risk factor for IHD in the previous study and is regarded as a minor risk factor for IHD, ${ }^{910}$ if only by its association with other risk factors such as hyperlipidaemia, hypertension, diabetes, and physical inactivity, ${ }^{11}$ yet this study has not found any relation that invites comment. Perhaps the pre-employment medical examination excluding notably overweight people from work as a gold miner has reduced the prevalence of obesity in this cohort and resulted in a loss of power of the statistical tests. To refute this argument is the fact that at the start of the study there was a large variation in the quetelet index (range 17 to 44 with mean (SD) $26 \cdot 8(4 \cdot 5))$. Hence this lack of a relation remains an unexplained feature of the data. The previous study ${ }^{3}$ examined the reliability of the ICD codes as given on the death certificate and used to calculate SMRs. Only $3 / 4$ of the codes given on the death certificates turned out to have been correct when these were further examined by GKS-C in the light of clinical notes and necropsy reports (table 3 ). Although these codes are accurate for traumatic deaths, they become much less so for other disease processes, although this accuracy is improved by broadening the disease class-for example, cancer instead of lung cancer. Ischaemic heart disease is so prevalent in this community, accounting for over $1 / 3$ of all deaths that the codes are accurate for this condition. There is very poor accuracy for conditions such as COPD, renal failure, cirrhosis of the liver, pneumonia, or tuberculosis, with about either $1 / 3$ wrongly coded or $1 / 3$ missed. This inaccuracy is in that segment of the South African population where one would expect the greatest accuracy due to the fact that they have had access to medical care and have been medically examined periodically for all their working lives.

We acknowledge Professor Pat Hessel for his helpful comments.

1 Liddell FD, McDonald JC, Thomas DC. Methods of cohort analysis: appraisal by application to asbestos $\mathrm{min}$ ing. Fournal of the Royal Statistical Society 1977;140: ing. four 91 .

2 Sluis-Cremer GK, Liddell FD, Logan WP, Bezuidenhout BN. The mortality of amphibole miners in South Africa BN. The mortality of amphibole miners in
1946-80. Br f Ind Med 1992;49:566-75.

3 Wyndham CH, Bezuidenhout BN, Greenacre M, SluisCremer GK. Mortality of middle aged white South African gold miners. $B r \mathcal{F}$ Ind Med 1986;43:677-84.

4 Breslow NE, Day NE. Statistical methods in cancer research. Vol 1. The analysis of case-control studies. Vol 2. The design and analysis of cohort studies. Lyon: International Agency for Research on Cancer, 1987.

5 Chekoway H, Pearce NE, Crawford-Brown DJ. Research methods in occupational epidemiology. Oxford: Oxford University Press, 1989. (Monographs in epidemiology and biostatistics. Vol 13.)

6 Wacholder S, Mclaughlin JK, Silverman DT, Mandel JS. Selection of controls in case-control studies. Am $\mathcal{f}$

7 Beadle DG. The relationship between the amount of dust breathed and the development of radiological signs of silicosis: an epidemiology study in South African gold miners. Presented at Johannesburg Pneumoconiosi Conference 1969. Inhaled Particles III. Vol 2. 953-64.

8 Page-Shipp RJ, Harris E. A study of the dust exposure of South African white gold miners. Fournal of the South African Institute of Minerals and Metallurgy 1972; 73:10-23.

9 Rossouw JE, Du Plessis JP, Benade AJ, Jordaan PCJ, Kotze JP, Jooste PL, Ferreira JJ. Coronary risk factor screening in three rural communities: The CORIS baseline study. $S$ Afr Med $\mathcal{f} 1983 ; 64: 430-6$.

10 Rossouw JE, Jooste PL, Steenkamp HJ, Thompson ML, Jordaan PCJ, Swanepoel ASP. Socio-economic status, risk factors and coronary heart disease. The CORIS baseline study. S Afr Med $\mathcal{F} 1990 ; 78: 82-5$.

11 The pooling project research group. Relationship of systolic blood pressure, serum cholesterol, smoking habit, relablood pressure, serum cholesterol, smoking habit, relative weight and ECG abnormalities to incidence of major coronary events: final report of the pooling project. $f$
Chron Dis 1978;31:201-306. 